\title{
TOTALLY GEODESIC SUBALGEBRAS IN 2-STEP NILPOTENT LIE ALGEBRAS
}

\author{
RACHELLE C. DECOSTE AND LISA DEMEYER
}

\begin{abstract}
We describe totally geodesic subalgebras of a metric 2-step nilpotent Lie algebra $\mathfrak{n}$. We prove that a totally geodesic subalgebra of $\mathfrak{n}$ is either abelian and flat or can be decomposed as a direct sum determined by the curvature transformation. In addition, we give conditions under which a totally geodesic submanifold of a simply connected 2-step nilpotent Lie group is a totally geodesic subgroup. We follow Eberlein's 1994 paper in which he imposes the condition of nonsingularity on $\mathfrak{n}$. We remove this restriction and illustrate the distinction between the nonsingular case and the unrestricted case.
\end{abstract}

1. Introduction. The purpose of this paper is to study totally geodesic submanifolds of a simply connected 2-step nilpotent Lie group $N$ equipped with a left invariant metric. Two-step nilpotent Lie groups are nonabelian Lie groups which are, in a sense, one step away from being abelian. Each simply connected 2-step nilpotent Lie group is diffeomorphic to $\mathbb{R}^{n}$ for some $n$. However, nonabelian, 2-step nilpotent Lie groups with a left invariant metric are not flat. Two-step nilpotent Lie groups are simple enough that many geometric quantities can be explicitly computed but complex enough as to provide interesting geometric results. See $[\mathbf{1}, \mathbf{3}, \mathbf{4}, \mathbf{5}, \mathbf{6}, \mathbf{7}, \mathbf{1 1}, \mathbf{1 2}]$, and others.

A subgroup of $N$ which passes through the identity, $e$, of $N$ is totally geodesic if it is totally geodesic at $e$. To study the totally geodesic subgroups through $e$ of $N$, it suffices to study the associated Lie algebra $\mathfrak{n}$. Note that not all totally geodesic submanifolds of $N$ are subgroups or even translates of totally geodesic subgroups by left translation.

Throughout the paper, we let $N$ denote a simply connected, 2-step nilpotent Lie group. Let $\mathfrak{n}$ denote the Lie algebra of $N$ with inner

2010 AMS Mathematics subject classification. Primary 53C30, 22 E25.

Keywords and phrases. Two-step nilpotent Lie algebra and totally geodesic subalgebra.

Received by the editors on August 23, 2013. 
product $\langle$,$\rangle . The inner product on \mathfrak{n}$ determines a left invariant metric on the Lie group $N$ and the geometry of the Lie group $N$ can be studied using the metric Lie algebra $\{\mathfrak{n},\langle\rangle$,$\} .$

In [5], Eberlein studies totally geodesic subalgebras and totally geodesic subgroups in nonsingular 2-step nilpotent Lie groups. A 2-step nilpotent Lie group is nonsingular if the Lie algebra has the property that, for all elements $X$ in $\mathfrak{n}-\mathfrak{z}$, ad $X$ maps surjectively onto the center. One of Eberlein's main results characterizes Heisenberg type Lie groups in terms of an abundance of three-dimensional totally geodesic subgroups which are isometric to the Heisenberg group, up to scaling of the metric by a positive constant. Heisenberg type groups are all, by definition, nonsingular. Gornet and Mast [7] introduced the notion of a Heisenberg-like nilpotent Lie algebra. The Heisenberg-like Lie algebras are defined by a similar abundance of three-dimensional, totally geodesic subalgebras used in [5] to characterize Heisenberg type Lie groups. However, Heisenberg-like Lie algebras are not necessarily nonsingular. Many of the results in [5] can be generalized nontrivially to the case where $N$ does not have the nonsingularity condition imposed by Eberlein. The results of [5] are summarized and discussed in [6], where it is noted that it is unknown if the hypothesis of nonsingularity used throughout [5] is necessary or merely convenient for the results in [5]. The study of the geometry of 2-step nilpotent Lie groups that may not be nonsingular, and in particular the investigation of totally geodesic subgroups and submanifolds of a 2-step nilpotent Lie group $N$, is the main focus of this paper.

The outline of the paper is as follows. In Section 2, we provide useful properties of 2-step nilpotent Lie groups and Lie algebras along with examples, which are referenced throughout the paper. In Section 3, we describe the curvature tensor on a metric, 2-step nilpotent Lie group. This follows [5]; however, it is done without the restriction that $N$ is nonsingular. The generalization presented here includes the singular case and extends Eberlein's results with only slight, and expected, changes. Section 3 contains results on the curvature tensor, and here we generalize two useful lemmas of Eberlein [5]. Section 4 contains the main results of this paper. Here we describe the totally geodesic subalgebras of $\mathfrak{n}$ in terms of the metric Lie algebra data. As in [5], we conclude that totally geodesic subgroups of $N$ are often abelian and flat, and that such subgroups are abundant in 2-step nilpotent 
$N$. In [5, Proposition 3.5], Eberlein shows that all totally geodesic subalgebras of nonsingular $\mathfrak{n}$ are either abelian and flat, or have a tangent space which decomposes in a simple way. In Theorem 4.10 below, we give a description of all totally geodesic subalgebras which are not abelian and flat in terms of the metric Lie algebra data from $\mathfrak{n}$, using a decomposition of the tangent space in terms of metric Lie algebra data. Here the decomposition is notably more complicated than in the nonsingular case. In addition, other useful criteria are established under which a subalgebra of $\mathfrak{n}$ is totally geodesic.

2. Preliminaries. Let $\{\mathfrak{n},\langle\rangle$,$\} be a 2$-step nilpotent Lie algebra with a left invariant metric. Let $\{N,\langle\rangle$,$\} be the simply-connected,$ 2 -step nilpotent metric Lie group with Lie algebra $\mathfrak{n}$. Let $\mathfrak{z}$ denote the center of $\{\mathfrak{n},\langle\rangle$,$\} , and define \mathfrak{v}$ as the orthogonal complement of $\mathfrak{z}$, $\mathfrak{v}=\mathfrak{z}^{\perp}$.

Definition 2.1. For each nonzero $Z \in \mathfrak{z}$, define a skew symmetric linear transformation $j(Z): \mathfrak{v} \rightarrow \mathfrak{v}$ by $\langle[X, Y], Z\rangle=\langle j(Z) X, Y\rangle$ for all $X, Y \in \mathfrak{v}$ and $Z \in \mathfrak{z}$.

We assume $j(Z)$ is not identically zero for any nonzero $Z \in \mathfrak{z}$. This is equivalent to assuming that $N$ has no Euclidean deRham factor.

We now prove the following useful lemma.

Lemma 2.2. Let $\{\mathfrak{n},\langle\rangle$,$\} be a 2-step nilpotent metric Lie algebra with$ no Euclidean deRham factor. Let $X, Y \in \mathfrak{v}$ and $Z \in \mathfrak{z}$.

(i) $[X, j(Z) X]=0$ if and only if $j(Z) X=0$.

(ii) $j([X, Y]) X=0$ if and only if $[X, Y]=0$.

(iii) $j(Z)^{2} X=0$ if and only if $j(Z) X=0$.

Proof.

(i) Assume $[X, j(Z) X]=0$ for some $X \in \mathfrak{v}$ and $Z \in \mathfrak{z}$. Then $\langle[X, j(Z) X], Z\rangle=|j(Z) X|^{2}=0$. Thus, $j(Z) X=0$. The other direction is obvious.

(ii) Assume $j([X, Y]) X=0$ for some $X, Y \in \mathfrak{v}$. Then $\langle j([X, Y]) X, Y\rangle$ $=|[X, Y]|^{2}=0$. Thus, $[X, Y]=0$. The other direction is obvious. 
(iii) Assume $j(Z)^{2} X=0$ for some $X \in \mathfrak{v}$ and $Z \in \mathfrak{z}$. Then $\left\langle j(Z)^{2} X, Y\right\rangle=0$ for all $Y \in \mathfrak{v}$. By the skew symmetry of $j(Z),-\langle j(Z) X, j(Z) Y\rangle=0$. Letting $Y=X$, we then have $\langle j(Z) X, j(Z) X\rangle=|j(Z) X|^{2}=0$. Finally, $j(Z) X=0$. The other direction is obvious.

The $j(Z)$ maps are used to define the Lie algebra as nonsingular, almost nonsingular or singular as follows.

Definition 2.3. Let $\{\mathfrak{n},\langle\rangle$,$\} denote a 2-step nilpotent metric Lie$ algebra.

(i) If $j(Z)$ is nonsingular for every nonzero $Z \in \mathfrak{z}$, then $\mathfrak{n}$ is said to be (strictly) nonsingular.

(ii) If $j(Z)$ is nonsingular for every $Z$ in an open dense subset of $\mathfrak{z}$, then $\mathfrak{n}$ is said to be almost nonsingular.

(iii) If $j(Z)$ is singular for all $Z \in \mathfrak{z}$, then $\mathfrak{n}$ is said to be (strictly) singular.

A 2-step nilpotent Lie group $N$ is defined to be nonsingular if its associated Lie algebra is nonsingular. The other cases follow.

Lemma 2.4 ([7], Lemma 1.16). Every 2-step nilpotent Lie group is precisely one of the following: nonsingular, almost nonsingular or singular.

We give some examples of the various classes of Lie algebras. These examples will be referred to later in the paper.

\section{Example 2.5.}

(i) ([7] Example 1.14). Let $\mathfrak{n}$ be a 2-step nilpotent Lie algebra with basis $\left\{X_{1}, X_{2}, X_{3}, X_{4}, Z_{1}, Z_{2}\right\}$. Define $j\left(Z_{1}\right)=A$ and $j\left(Z_{2}\right)=B$ as

$$
A=\left(\begin{array}{cccc}
0 & -1 & 0 & 0 \\
1 & 0 & 0 & 0 \\
0 & 0 & 0 & 0 \\
0 & 0 & 0 & 0
\end{array}\right), \quad B=\left(\begin{array}{cccc}
0 & 0 & 0 & 0 \\
0 & 0 & 0 & 0 \\
0 & 0 & 0 & -1 \\
0 & 0 & 1 & 0
\end{array}\right) .
$$


This is an almost nonsingular Lie algebra. If $Z=\alpha_{1} Z_{1}+\alpha_{2} Z_{2} \in \mathfrak{z}$, then $j(Z)$ has eigenvalues $\left\{ \pm i \alpha_{1}, \pm i \alpha_{2}\right\}$, so $j(Z)$ is nonsingular on $\mathcal{O}=\left\{\alpha_{1} Z_{1}+\alpha_{2} Z_{2} \in \mathfrak{z} \mid \alpha_{1} \neq 0, \alpha_{2} \neq 0\right\}$.

(ii) The previous example generalizes to a 2 -step nilpotent Lie algebra $\mathfrak{n}$ of dimension $4 k+2 k$ with basis $\left\{X_{1}, \ldots, X_{4 k}, Z_{1}, \ldots, Z_{2 k}\right\}$. The matrices $A$ and $B$ above are used as $k$ diagonal blocks to define the $j\left(Z_{l}\right)$ maps, $l=1, \ldots, 2 k$. For instance, with $k=2$, and letting 0 represent the $4 \times 4$ zero matrix, the maps are represented by

$$
\begin{array}{lll}
j\left(Z_{1}\right)=\left(\begin{array}{cc}
A & 0 \\
0 & 0
\end{array}\right), & j\left(Z_{2}\right)=\left(\begin{array}{cc}
B & 0 \\
0 & 0
\end{array}\right), \\
j\left(Z_{3}\right)=\left(\begin{array}{cc}
0 & 0 \\
0 & A
\end{array}\right), & j\left(Z_{4}\right)=\left(\begin{array}{cc}
0 & 0 \\
0 & B
\end{array}\right) .
\end{array}
$$

This defines $\left[X_{1}, X_{2}\right]=-\left[X_{2}, X_{1}\right]=Z_{1},\left[X_{3}, X_{4}\right]=-\left[X_{4}, X_{3}\right]=$ $Z_{2},\left[X_{5}, X_{6}\right]=-\left[X_{6}, X_{5}\right]=Z_{3},\left[X_{7}, X_{8}\right]=-\left[X_{8}, X_{7}\right]=Z_{4}$ and all other brackets zero.

In the general case, $Z=\alpha_{1} Z_{1}+\cdots+\alpha_{2 k} Z_{2 k}$ has eigenvalues $\left\{ \pm i \alpha_{l} \mid 1 \leq l \leq 2 k\right\}$, and therefore $j(Z)$ is nonsingular on $\mathcal{O}=\left\{\alpha_{1} Z_{1}+\cdots+\alpha_{2 k} Z_{2 k} \in \mathfrak{z} \mid \alpha_{l} \neq 0,1 \leq l \leq 2 k\right\} ;$ thus, the Lie algebra $\mathfrak{n}$ is almost nonsingular.

(iii) This example is a generalization of [4, Example 4.8]. Let $\mathfrak{n}$ be a ten-dimensional 2-step nilpotent Lie algebra with basis $\left\{X_{1}, \ldots, X_{6}, Z_{1}, \ldots, Z_{4}\right\}$. Define

$$
\begin{aligned}
j\left(Z_{1}\right)=\left(\begin{array}{cccccc}
0 & -1 & 0 & 0 & 0 & 0 \\
1 & 0 & 0 & 0 & 0 & 0 \\
0 & 0 & 0 & 0 & 0 & 0 \\
0 & 0 & 0 & 0 & 0 & 0 \\
0 & 0 & 0 & 0 & 0 & 0 \\
0 & 0 & 0 & 0 & 0 & 0
\end{array}\right), \quad j\left(Z_{2}\right)=\left(\begin{array}{cccccc}
0 & 0 & -1 & 0 & 0 & 0 \\
0 & 0 & 0 & 0 & 0 & 0 \\
1 & 0 & 0 & 0 & 0 & 0 \\
0 & 0 & 0 & 0 & 0 & 0 \\
0 & 0 & 0 & 0 & 0 & 0 \\
0 & 0 & 0 & 0 & 0 & 0
\end{array}\right) \\
j\left(Z_{3}\right)=\left(\begin{array}{ccccccc}
0 & 0 & 0 & 0 & 0 & 0 \\
0 & 0 & 0 & 0 & 0 & 0 \\
0 & 0 & 0 & 0 & 0 & 0 \\
0 & 0 & 0 & 0 & -1 & 0 \\
0 & 0 & 0 & 1 & 0 & 0 \\
0 & 0 & 0 & 0 & 0 & 0
\end{array}\right), \quad j\left(Z_{4}\right)=\left(\begin{array}{cccccc}
0 & 0 & 0 & 0 & 0 & 0 \\
0 & 0 & 0 & 0 & 0 & 0 \\
0 & 0 & 0 & 0 & 0 & 0 \\
0 & 0 & 0 & 0 & 0 & -1 \\
0 & 0 & 0 & 0 & 0 & 0 \\
0 & 0 & 0 & 1 & 0 & 0
\end{array}\right) .
\end{aligned}
$$

This defines brackets $\left[X_{1}, X_{2}\right]=-\left[X_{2}, X_{1}\right]=Z_{1},\left[X_{1}, X_{3}\right]=$ $-\left[X_{3}, X_{1}\right]=Z_{2},\left[X_{4}, X_{5}\right]=-\left[X_{5}, X_{4}\right]=Z_{3}$ and $\left[X_{4}, X_{6}\right]=$ $-\left[X_{6}, X_{4}\right]=Z_{4}$, with all other brackets zero. This is a singular 
Lie algebra. If $Z=\alpha_{1} Z_{1}+\alpha_{2} Z_{2}+\alpha_{3} Z_{3}+\alpha_{4} Z_{4} \in \mathfrak{z}$, then $j(Z)$ has eigenvalues $\left\{0, \pm i \sqrt{\alpha_{1}^{2}+\alpha_{2}^{2}}, \pm i \sqrt{\alpha_{3}^{2}+\alpha_{4}^{2}}\right\}$ and is clearly singular. Further generalization of this example allows the creation of a singular 2-step nilpotent Lie algebra of dimension $k(n+1)+n$ for any positive integers $k$ and $n$, as in [2, Example 6.3].

3. Curvature transformations. Let $\nabla_{\xi_{1}} \xi_{2}$ denote the covariant derivative on $\mathfrak{n}$, the 2-step nilpotent Lie algebra $\mathfrak{n}=\mathfrak{v} \oplus \mathfrak{z}$, where $\xi_{1}, \xi_{2}$ are left invariant vector fields on the corresponding Lie group $N$. As in $[\mathbf{5}, 1.10]$, we have the following formulas:

$$
\begin{aligned}
& \nabla_{X} Y=\frac{1}{2}[X, Y] \text { for all } X, Y \in \mathfrak{v}, \\
& \nabla_{X} Z=-\frac{1}{2} j(Z) X \text { for all } X \in \mathfrak{v}, Z \in \mathfrak{z}, \\
& \nabla_{Z_{1}} Z_{2}=0 \text { for all } Z_{1}, Z_{2} \in \mathfrak{z} .
\end{aligned}
$$

Recall the curvature tensor is defined as $R\left(\xi_{1}, \xi_{2}\right) \xi_{3}=-\nabla_{\left[\xi_{1}, \xi_{2}\right]} \xi_{3}+$ $\nabla_{\xi_{1}}\left(\nabla_{\xi_{2}} \xi_{3}\right)-\nabla_{\xi_{2}}\left(\nabla_{\xi_{1}} \xi_{3}\right)$ for all left invariant vector fields $\xi_{1}, \xi_{2}$ and $\xi_{3}$ on $N$. It is straightforward to compute the following (cf., [5, 1.11]) for all $X, Y, V \in \mathfrak{v}$ and $Z, Z_{1}, Z_{2}, Z_{3} \in \mathfrak{z}$.

$$
\begin{aligned}
R(X, Y) V & =\frac{1}{2} j([X, Y]) V-\frac{1}{4} j([Y, V]) X+\frac{1}{4} j([X, V]) Y, \\
R\left(X, Z_{1}\right) Z_{2} & =-\frac{1}{4} j\left(Z_{1}\right) j\left(Z_{2}\right) X, \\
R(X, Z) Y & =-\frac{1}{4}[X, j(Z) Y], \\
R\left(Z_{1}, Z_{2}\right) Z_{3} & =0 .
\end{aligned}
$$

For each $\xi \in \mathfrak{n}$, let $R_{\xi}$ be the curvature transformation defined on $\mathfrak{n}$ by $R_{\xi}(\eta)=R(\eta, \xi) \xi$. The following two results extend [5, Lemma A] to include the singular case.

Lemma 3.1. Let $\{\mathfrak{n},\langle\rangle$,$\} be a 2-step nilpotent metric Lie algebra.$ Then, for nonzero $\xi \in \mathfrak{n}, \mathfrak{v}$ and $\mathfrak{z}$ are invariant subspaces of $R_{\xi}$. Moreover,

(i) If $X \in \mathfrak{v}$ is nonzero, then $R_{X}$ is negative semidefinite on $\mathfrak{v}$ and positive semidefinite on $\mathfrak{z}$.

(ii) If $Z \in \mathfrak{z}$ is nonzero, then $R_{Z}$ is positive semidefinite on $\mathfrak{v}$ and zero on $\mathfrak{z}$. 
(iii) If $\xi=X+Z \in \mathfrak{n}$ is nonzero, then $R_{\xi}$ is positive semidefinite on $\mathfrak{z}$.

Proof. The proofs of (i) and (ii) are identical to the proof of [5, Lemma A] with the exception that $\frac{1}{4}|j(Z) X|^{2} \geq 0$ since $j(Z)$ can be singular, resulting in the semidefiniteness. The proof of (iii) follows from (i) and (ii).

Lemma 3.2 below, a generalization of [5, Lemma A], is used to give a decomposition of the tangent space of a totally geodesic submanifold of a 2-step nilpotent Lie algebra in Section 4. In [5, Lemma A], if $\xi$ is in either $\mathfrak{v}$ or $\mathfrak{z}$ for a nonsingular Lie algebra $\mathfrak{n}=\mathfrak{v} \oplus \mathfrak{z}$, then the eigenvectors of $R_{\xi}$ were easily seen to lie in either $\mathfrak{v}$ or $\mathfrak{z}$. In the singular case, the presence of zero eigenvalues is an added complication. As seen in this result, a vector of the zero eigenspace must satisfy certain conditions related to $\xi$.

Lemma 3.2. Let $\{\mathfrak{n},\langle\rangle$,$\} be a 2-step nilpotent metric Lie algebra.$ Then

(i) For $X \in \mathfrak{v}$, let $\sigma$ be an eigenvector of $R_{X}$. If $\sigma$ corresponds to a nonzero eigenvalue, then $\sigma$ lies in $\mathfrak{v}$ or $\mathfrak{z}$. If $\sigma$ corresponds to the zero eigenvalue, then $\sigma=\sigma_{\mathfrak{v}}+\sigma_{\mathfrak{z}}, \sigma_{\mathfrak{v}} \in \mathfrak{v}, \sigma_{\mathfrak{z}} \in \mathfrak{z}$, where $\left[X, \sigma_{\mathfrak{v}}\right]=0$ and $j\left(\sigma_{\mathfrak{z}}\right) X=0$.

(ii) For $Z \in \mathfrak{z}$, let $\sigma$ be an eigenvector of $R_{Z}$. If $\sigma$ corresponds to a nonzero eigenvalue, then $\sigma$ lies in $\mathfrak{v}$. If $\sigma$ corresponds to the zero eigenvalue, then $\sigma=\sigma_{\mathfrak{v}}+\sigma_{\mathfrak{z}}, \sigma_{\mathfrak{v}} \in \mathfrak{v}, \sigma_{\mathfrak{z}} \in \mathfrak{z}$, where $\sigma_{\mathfrak{v}} \in \operatorname{ker} j(Z)$.

\section{Proof.}

(i) Let $X \in \mathfrak{v}$, and let $\sigma$ be an eigenvector of $R_{X}$ such that $R_{X}(\sigma)=$ $\lambda \sigma, \lambda \neq 0$. Since $R_{X}$ is negative semidefinite on $\mathfrak{v}$ and positive semidefinite on $\mathfrak{z}$ by Lemma 3.1(i), it follows that $\sigma$ lies in $\mathfrak{v}$ or $\mathfrak{z}$. Now let $\sigma$ be an eigenvector of $R_{X}$ such that $R_{X}(\sigma)=0$. Write $\sigma=\sigma_{\mathfrak{v}}+\sigma_{\mathfrak{z}}, \sigma_{\mathfrak{v}} \in \mathfrak{v}$ and $\sigma_{\mathfrak{z}} \in \mathfrak{z}$. Then $0=R_{X}(\sigma)=$ $R_{X}\left(\sigma_{\mathfrak{v}}+\sigma_{\mathfrak{z}}\right)=R_{X}\left(\sigma_{\mathfrak{v}}\right)+R_{X}\left(\sigma_{\mathfrak{z}}\right)=-\frac{3}{4} j\left(\left[X, \sigma_{\mathfrak{v}}\right]\right) X+\frac{1}{4}\left[X, j\left(\sigma_{\mathfrak{z}}\right) X\right]$. Since $j\left(\left[X, \sigma_{\mathfrak{v}}\right]\right) X \in \mathfrak{v}$ and $\left[X, j\left(\sigma_{\mathfrak{z}}\right) X\right] \in \mathfrak{z}, j\left(\left[X, \sigma_{\mathfrak{v}}\right]\right) X=0$ and $\left[X, j\left(\sigma_{\mathfrak{z}}\right) X\right]=0$. Thus, by Lemma $2.2,\left[X, \sigma_{\mathfrak{v}}\right]=0$ and $j\left(\sigma_{\mathfrak{z}}\right) X=0$. 
(ii) Let $Z \in \mathfrak{z}$, and let $\sigma$ be an eigenvector of $R_{Z}$ such that $R_{Z}(\sigma)=$ $\lambda \sigma, \lambda \neq 0$. By Lemma 3.1(ii) $\sigma$ lies in $\mathfrak{v}$. Now let $\sigma$ be an eigenvector of $R_{Z}$ such that $R_{Z}(\sigma)=0$. Write $\sigma=\sigma_{\mathfrak{v}}+\sigma_{\mathfrak{z}}, \sigma_{\mathfrak{v}} \in \mathfrak{v}$ and $\sigma_{\mathfrak{z}} \in \mathfrak{z}$. Then $0=R_{Z}(\sigma)=R_{Z}\left(\sigma_{\mathfrak{v}}+\sigma_{\mathfrak{z}}\right)=R_{Z}\left(\sigma_{\mathfrak{v}}\right)+R_{Z}\left(\sigma_{\mathfrak{z}}\right)=$ $-\frac{1}{4} j(Z)^{2} \sigma_{\mathfrak{v}}$. Thus, $j(Z) \sigma_{\mathfrak{v}}=0$ by Lemma 2.2 .

Lemma B in [5] describes the Lie algebras of Heisenberg type with respect to the curvature transformations. Since Lie algebras of Heisenberg type must be nonsingular, there is no analogous result to be found here. However, a natural extension of Heisenberg type Lie algebras are Heisenberg-like Lie algebras, which are studied extensively in $[\mathbf{2}, \mathbf{7}]$ and discussed in more detail in subsection 4.1.

\section{Totally geodesic submanifolds and subalgebras.}

Definition 4.1. A submanifold $\bar{M}$ of a Riemannian manifold $M$ is said to be a totally geodesic submanifold if every geodesic of $\bar{M}$ is also a geodesic of $M$.

Equivalently, recall that a submanifold $\bar{M}$ of a manifold $M$ is totally geodesic if and only if the second fundamental form is identically zero on $\bar{M}$.

Definition 4.2. A subalgebra $\mathcal{H}$ of a metric Lie algebra is said to be a totally geodesic subalgebra if $\nabla_{\sigma} \xi \in \mathcal{H}$ for all $\sigma, \xi \in \mathcal{H}$.

A totally geodesic subgroup $H$ of a Lie group $G$ with left invariant metric is a Lie subgroup which is also totally geodesic as a submanifold. The subgroup $H$ is a totally geodesic subgroup if and only if the corresponding subalgebra $\mathcal{H}$ is a totally geodesic subalgebra. Not all totally geodesic submanifolds in a Lie group must be open sets of $g H$ where $H$ is a totally geodesic subgroup and $g$ is an element of the group. For example, not all geodesics are one-parameter subgroups. If $\gamma$ is a geodesic with $\gamma(0)=e$ and $\gamma^{\prime}(0)=X_{0}+Z_{0}$, then $\gamma$ is a one-parameter subgroup if and only if $j\left(Z_{0}\right) X_{0}=0$. See [4, Example 1 of (2.11)].

Given a totally geodesic subalgebra $\mathcal{H}$ of $\mathfrak{n}$, we describe the structure of $\mathcal{H}$. First we include a useful lemma and some examples. 
Lemma 4.3. Let $\mathcal{H}$ be a totally geodesic subalgebra of a 2-step nilpotent Lie algebra $\{\mathfrak{n},\langle\rangle$,$\} . If X+Z \in \mathcal{H}$, then $j(Z) X \in \mathcal{H} \cap \mathfrak{v}$.

Proof. Let $\xi=X+Z \in \mathcal{H}$. Since $\mathcal{H}$ is totally geodesic, $\nabla_{\xi} \xi \in \mathcal{H}$. Thus, $\nabla_{\xi} \xi=-j(Z) X \in \mathcal{H} \cap \mathfrak{v}$.

\section{Example 4.4.}

(i) [5]. Let $\mathcal{H}$ be an abelian subspace of $\mathfrak{v}$. Then $\mathcal{H}$ is a totally geodesic subalgebra.

(ii) [5]. Let $\mathcal{H}$ be a subspace of $\mathfrak{z}$. Then $\mathcal{H}$ is a totally geodesic subalgebra.

(iii) Consider $\mathfrak{n}$ of Example 2.5 (iii). Let $\mathcal{H}$ be the subspace of $\mathfrak{n}$ with basis $\left\{X_{1}, X_{2}, Z_{1}, X_{5}+Z_{4}\right\}$. This is a totally geodesic subalgebra.

(iv) Consider $\mathfrak{n}$ of Example 2.5 (iii). Let $\mathcal{H}$ be the subspace of $\mathfrak{n}$ with basis $\left\{X_{1}, X_{2}, Z_{1}, \alpha_{1} X_{5}+\alpha_{2} X_{6}, \alpha_{3} Z_{3}+\alpha_{4} Z_{4}\right\} . \mathcal{H}$ a is totally geodesic subalgebra if and only if $\alpha_{1} \alpha_{3}=-\alpha_{2} \alpha_{4}$.

Observe that the first two examples above are abelian and flat. In subsection 4.2 , we look more closely at totally geodesic subalgebras which are abelian and flat and say what we can about those that are not. In subsection 4.1, we continue to look at more examples which illustrate the importance of totally geodesic subalgebras.

4.1. Heisenberg type and Heisenberg-like Lie algebras. Heisenberg type and Heisenberg-like Lie algebras are classes of Lie algebras with an abundance of totally geodesic subalgebras. A 2-step nilpotent metric Lie algebra $\{\mathfrak{n},\langle\rangle$,$\} is defined to be of Heisenberg type if$ $j(Z)^{2}=-\left.|Z|^{2} I d\right|_{\mathfrak{v}}$ for every $Z \in \mathfrak{z}$. A simply connected 2-step nilpotent metric Lie group $N$ is of Heisenberg type if its Lie algebra $\mathfrak{n}$ is of Heisenberg type. Lie groups of Heisenberg type are a generalization of the well-studied Heisenberg group. Groups of Heisenberg type were introduced by Kaplan in [8], and their geometry has been studied extensively, including in $[\mathbf{4}, \mathbf{5}, \mathbf{9}]$. Heisenberg type Lie algebras are nonsingular.

If $\mathfrak{n}$ is a Heisenberg type Lie algebra, by [5, Example 3.4 (3)], $\mathcal{H}=\operatorname{span}\{X, Z, j(Z) X\}$ is a totally geodesic subalgebra of $\mathfrak{n}$ where $X \in \mathfrak{v}$ and $Z \in \mathfrak{z}$ are arbitrary nonzero vectors. Further, $H=\exp (\mathcal{H})$ 
is isometric to the standard metric three-dimensional Heisenberg group when the metric on $\mathcal{H}$ is multiplied by an appropriate positive constant.

In [5, Section 6], Eberlein also proves that every geodesic in a Heisenberg type simply-connected, nonsingular, 2-step nilpotent Lie group with a left invariant metric is contained in a three-dimensional, totally geodesic submanifold. Conversely, he shows that if $\{N,\langle\rangle\}$, a simply-connected, nonsingular, 2-step nilpotent Lie group with a left invariant metric, satisfies two conditions, then $N$ is of Heisenberg type. One condition is that $\mathcal{H} \cap \mathfrak{z} \neq\{0\}$. The second condition is that, for each geodesic $\gamma \in N$ with $\gamma(0)=e$, there exists a connected, three-dimensional totally geodesic submanifold $H$ such that $\gamma^{\prime}(0) \in \mathcal{H}=T_{e} H$. If $N$ has a one-dimensional center, the condition $\mathcal{H} \cap \mathfrak{z} \neq\{0\}$ can be removed, as in [5, Theorem 6.2].

Heisenberg-like Lie algebras are defined by the abundance of totally geodesic subalgebras, generalizing Lie algebras of Heisenberg type. Specifically, Gornet and Mast [7] define Heisenberg-like Lie algebras to be those 2-step, nilpotent Lie algebras with the property that $\operatorname{span}\left\{X_{m}, Z, j(Z) X_{m}\right\}$ is a totally geodesic subalgebra for every $Z \in \mathfrak{z}$ and every $X_{m} \in W_{m}(Z)$. Here $W_{m}(Z)$ is the invariant subspace of $j(Z)$ corresponding to $\vartheta_{m}(Z)$ where $\left\{ \pm i \vartheta_{m}\right\}$ is the set of eigenvalues of $j(Z)$; see [2] for further explanation. A 2-step nilpotent metric Lie group is Heisenberg-like if and only if its Lie algebra is Heisenberglike. By [7, Theorem 3.6], a Heisenberg-like metric Lie algebra is either strictly nonsingular or strictly singular. In [2], we construct an infinite family of examples of singular Heisenberg-like Lie algebras. Thus, these provide examples for which the results of [5] pertaining to totally geodesic subalgebras do not hold.

\subsection{Totally geodesic subalgebras which are abelian and flat.}

Lemma 4.5. Let $\{\mathfrak{n},\langle\rangle$,$\} be a 2-step nilpotent Lie algebra, and let \mathcal{H}$ be a subalgebra of $\mathfrak{n}$. If $\mathcal{H}$ is flat then $\mathcal{H}$ is abelian.

Proof. If $\mathcal{H}$ is a flat subalgebra of $\mathfrak{n}$, then $\nabla_{\xi} \sigma=0$ for all $\xi, \sigma \in \mathcal{H}$. Write $\xi=X+Z_{1}$ and $\sigma=Y+Z_{2} \in \mathcal{H}$, where $X, Y \in \mathfrak{v}$ and $Z_{1}, Z_{2} \in \mathfrak{z}$. Then,

$$
0=\nabla_{\xi} \sigma=\frac{1}{2}[X, Y]-\frac{1}{2} j\left(Z_{2}\right) X-\frac{1}{2} j\left(Z_{1}\right) Y
$$


Since $\mathfrak{n}=\mathfrak{v} \oplus \mathfrak{z},[X, Y] \in \mathfrak{z}$ and $-\frac{1}{2} j\left(Z_{2}\right) X-\frac{1}{2} j\left(Z_{1}\right) Y \in \mathfrak{v}$, we conclude that $[X, Y]=0$. Hence, $[\xi, \sigma]=0$ for any $\sigma, \xi \in \mathcal{H}$. Thus, $\mathcal{H}$ is abelian.

If $\mathcal{H}$ is totally geodesic, then abelian implies flat, as in the following lemma.

Lemma 4.6. Let $\{\mathfrak{n},\langle\rangle$,$\} be a 2-step nilpotent Lie algebra, and let \mathcal{H}$ be a totally geodesic subalgebra of $\mathfrak{n}$. If $\mathcal{H}$ is abelian, then $\mathcal{H}$ is flat.

Proof. Since $\mathcal{H}$ is totally geodesic, $\nabla_{\xi} \sigma \in \mathcal{H}$ for all $\xi, \sigma \in \mathcal{H}$. Again, write $\xi=X+Z_{1}$ and $\sigma=Y+Z_{2} \in \mathcal{H}$, where $X, Y \in \mathfrak{v}$ and $Z_{1}, Z_{2} \in \mathfrak{z} . \mathcal{H}$ is abelian; thus, $\nabla_{\xi} \sigma=\frac{1}{2}[X, Y]-\frac{1}{2} j\left(Z_{2}\right) X-\frac{1}{2} j\left(Z_{1}\right) Y=$ $-\frac{1}{2} j\left(Z_{2}\right) X-\frac{1}{2} j\left(Z_{1}\right) Y \in \mathcal{H}$. If $\mathcal{H}$ is not flat, $\nabla_{\xi} \sigma \neq 0$ for some $\xi, \sigma \in \mathcal{H}$. Then $-\frac{1}{2} j\left(Z_{2}\right) X-\frac{1}{2} j\left(Z_{1}\right) Y=V \in \mathcal{H}$ where $V \neq 0$. Consequently, $\left\langle j\left(Z_{2}\right) X, V\right\rangle+\left\langle j\left(Z_{1}\right) Y, V\right\rangle=\left\langle[X, V], Z_{2}\right\rangle+\left\langle[Y, V], Z_{1}\right\rangle \neq 0$, which contradicts the fact that $\mathcal{H}$ is abelian. Thus $\mathcal{H}$ must also be flat.

Here, and throughout the remainder of the paper, $\pi_{\mathfrak{v}}(\mathcal{H})$ and $\pi_{\mathfrak{z}}(\mathcal{H})$ represent the projections of $\mathcal{H}$ onto $\mathfrak{v}$ and $\mathfrak{z}$, respectively. In the nonsingular case, it is true that $\pi_{\mathfrak{v}}(\mathcal{H})=\mathcal{H} \cap \mathfrak{v}$ and $\pi_{\mathfrak{z}}(\mathcal{H})=\mathcal{H} \cap \mathfrak{z}$; however, in general $\pi_{\mathfrak{v}}(\mathcal{H}) \neq \mathcal{H} \cap \mathfrak{v}$ and $\pi_{\mathfrak{z}}(\mathcal{H}) \neq \mathcal{H} \cap \mathfrak{z}$.

The following is an example of a singular 2-step nilpotent metric Lie algebra with an infinite family of two-dimensional, flat, abelian, totally geodesic subalgebras.

Example 4.7. Let $\mathfrak{n}$ be the singular, metric, 2-step nilpotent Lie algebra with orthonormal basis $\left\{V, X, Y, Z_{1}, Z_{2}\right\}$ and bracket relation given by $[X, Y]=Z_{1}$ and $[V, X]=Z_{2}$. Let $\mathcal{H}=\operatorname{span}\left\{\alpha_{1} V+\right.$ $\left.\alpha_{2} Y, \alpha_{1} Z_{1}+\alpha_{2} Z_{2}\right\}$, where $\alpha_{1}, \alpha_{2} \neq 0$. It is straightforward to show that $\mathcal{H}$ is flat, abelian and totally geodesic. Note that $j\left(Z_{1}\right) Y \notin \mathcal{H}$ even though $Z_{1} \in \pi_{\mathfrak{z}}(\mathcal{H})$ and $Y \in \pi_{\mathfrak{v}}(\mathcal{H})$.

In [5], Propositions 3.5 and 3.7 describe necessary and sufficient conditions for a subalgebra $\mathcal{H}$ of nonsingular $\mathfrak{n}$ to be totally geodesic. The main result of this paper, Theorem 4.10, is the generalized result of the first proposition when singularity is allowed. Proposition 4.21 is the generalization of the other direction. The following proposition 
is necessary in the proof of our theorem. Following its proof, we state Eberlein's result and then the generalization.

Proposition 4.8. Let $\mathcal{H}$ be a totally geodesic subalgebra of a 2-step nilpotent metric Lie algebra $\{\mathfrak{n},\langle\rangle$,$\} .$

(i) If $\mathcal{H} \cap \mathfrak{z}=\{0\}$, then $\mathcal{H}$ is abelian and flat.

(ii) If $\mathcal{H} \cap \mathfrak{v}=\{0\}$, then $\mathcal{H}$ is abelian and flat.

Proof. Let $\xi=X+Z_{1}, \sigma=Y+Z_{2} \in \mathcal{H}$, where $X, Y \in \mathfrak{v}$ and $Z_{1}, Z_{2} \in \mathfrak{z}$. Since $\mathcal{H}$ is a subalgebra, $[\xi, \sigma]=[X, Y] \in \mathcal{H}$. So $[X, Y] \in \mathcal{H} \cap \mathfrak{z}$.

Then, since $\mathcal{H}$ is totally geodesic, $\nabla_{\xi} \sigma \in \mathcal{H}$. Hence, $\nabla_{\xi} \sigma=$ $\frac{1}{2}[X, Y]-\frac{1}{2} j\left(Z_{2}\right) X-\frac{1}{2} j\left(Z_{1}\right) Y \in \mathcal{H}$. Since $[X, Y] \in \mathcal{H} \cap \mathfrak{z}$, we have $\left(j\left(Z_{1}\right) Y+j\left(Z_{2}\right) X\right) \in \mathcal{H} \cap \mathfrak{v}$.

(i) If $\mathcal{H} \cap \mathfrak{z}=\{0\}$, then $[\xi, \sigma]=0$. Hence, $\mathcal{H}$ is abelian and then flat by Lemma 4.6 .

(ii) Since $\mathcal{H}$ is a subalgebra, $[\xi, \sigma]=[X, Y] \in \mathcal{H} \cap \mathfrak{z}$ for all $\xi, \sigma \in \mathcal{H}$ as above. We show $[X, Y]=0$ when $\mathcal{H} \cap \mathfrak{v}=\{0\}$. Since $\mathcal{H}$ is totally geodesic, $\nabla_{[X, Y]}\left(X+Z_{1}\right)=\nabla_{[X, Y]} X=-\frac{1}{2} j([X, Y]) X \in \mathcal{H}$; thus, $j([X, Y]) X \in \mathcal{H} \cap \mathfrak{v}=\{0\}$. By Lemma $2.2,[X, Y]=0$. Therefore, $\mathcal{H}$ is abelian; flat again follows by Lemma 4.6.

In Proposition 4.9 we restate Propositions 3.5 and 3.7 from [5], which include the assumption of nonsingularity.

Proposition 4.9. [5, Propositions 3.5, 3.7]. Let $\{N,\langle\rangle$,$\} be a simply$ connected, nonsingular, 2-step nilpotent Lie group with a left invariant metric. Let $\mathcal{H}$ be a totally geodesic subalgebra of $\mathfrak{n}$. Then, exactly one of the following occurs:

(i) $\mathcal{H}$ is an abelian subspace of $\mathfrak{v}$ and $\mathcal{H}$ is flat.

(ii) $\mathcal{H}$ is a subspace of $\mathfrak{z}$ and $\mathcal{H}$ is flat.

(iii) (a) $\mathcal{H}$ is the direct sum of the nonzero subspaces $\mathcal{H} \cap \mathfrak{v}$ and $\mathcal{H} \cap \mathfrak{z}$.

(b) $\mathcal{H} \cap \mathfrak{v}$ is invariant under $j(Z)$ for every $Z \in \mathcal{H} \cap \mathfrak{z}$.

Moreover, if $\mathcal{H}$ is a subalgebra of $\mathfrak{n}$ that satisfies one of the conditions above, then $\mathcal{H}$ is totally geodesic. 
In the general case, where the condition of nonsingularity is removed, the tangent space does not decompose as a direct sum of the subspaces $\mathcal{H} \cap \mathfrak{v}$ and $\mathcal{H} \cap \mathfrak{z}$. In this case, we conclude that, if $\mathcal{H}$ is a totally geodesic subalgebra, it is either abelian and flat or it has a tangent space which can be decomposed in terms of the eigenspaces of the curvature transformation. The proof of this result, stated in Theorem 4.10, is found at the end of this section.

Let $H$ be a totally geodesic submanifold of $N$ containing the identity $e$, and let $\mathcal{H}=T_{e} H \subseteq \mathfrak{n}$. For any element $\xi \in \mathcal{H}$, define $\mathcal{H}_{+}^{\xi}=\left\{\sigma \in \mathcal{H} \mid R_{\xi}(\sigma)=\lambda \sigma, \lambda>0\right\}, \mathcal{H}_{-}^{\xi}=\left\{\sigma \in \mathcal{H} \mid R_{\xi}(\sigma)=\lambda \sigma, \lambda<0\right\}$ and $\mathcal{H}_{0}^{\xi}=\left\{\sigma \in \mathcal{H} \mid R_{\xi}(\sigma)=0\right\}$.

Theorem 4.10. Let $\{N,\langle\rangle$,$\} be a simply connected 2-step nilpotent$ Lie group with a left invariant metric. Let $\mathcal{H}$ be a totally geodesic subalgebra of $\mathfrak{n}$. Then $\mathcal{H} \cap \mathfrak{v}$ is invariant under $j(Z)$ for every $Z \in \mathcal{H} \cap \mathfrak{z}$ and exactly one of the following occurs:

(i) $\mathcal{H}$ is abelian and flat.

(ii) $\mathcal{H}$ is the direct sum of nonzero subspaces $\mathcal{H}_{+}^{Z}$ and $\mathcal{H}_{0}^{Z}$ for some $Z \in \mathcal{H} \cap \mathfrak{z}$.

4.3. Criteria for a subalgebra to be totally geodesic. In [5], Eberlein proved the following two lemmas under the hypothesis that $\mathfrak{n}$ is nonsingular. The results hold in the general case, without the nonsingularity assumption with no change to the proofs.

Lemma 4.11. [5, Lemma 2.1]. Let $\{N,\langle\rangle$,$\} be a simply-connected,$ 2-step nilpotent Lie group with a left invariant metric. Let $H$ be a totally geodesic submanifold of $N$ that contains the identity, and let $\mathcal{H}=T_{e} H \subseteq T_{e} N=\mathfrak{n}$. If $\xi_{1}, \xi_{2}$ and $\xi_{3}$ are arbitrary elements of $\mathcal{H}$, then $R\left(\xi_{1}, \xi_{2}\right) \xi_{3} \in \mathcal{H}$, where $R$ is the curvature tensor of $\mathfrak{n}$.

Lemma 4.12. [5, Lemma 2.2 (b), (c)]. Let $H$ be a totally geodesic submanifold of $N$ that contains the identity e, and let $\mathcal{H}=T_{e} H \subseteq \mathfrak{n}$. If $\mathcal{H} \cap \mathfrak{v} \neq 0$ or $\mathcal{H} \cap \mathfrak{z} \neq 0$, then

(i) $\mathcal{H} \cap \mathfrak{v}$ is invariant under $j(Z)^{2}$ for every $Z \in \mathcal{H} \cap \mathfrak{z}$,

(ii) $[X, j(Z) Y] \in \mathcal{H} \cap \mathfrak{z}$ for any elements $X, Y \in \mathcal{H} \cap \mathfrak{v}$ and $Z \in \mathcal{H} \cap \mathfrak{z}$. 
Eberlein also proved the following under the assumption that $\mathfrak{n}$ is nonsingular. The following lemma does not hold if $\mathfrak{n}$ is not nonsingular, as we illustrate below.

Lemma 4.13. [5, Lemma 2.2 (a), (d)]. Let $H$ be a totally geodesic submanifold of a 2-step nilpotent nonsingular Lie group $N$. Assume $H$ contains the identity $e$, and let $\mathcal{H}=T_{e} H \subseteq \mathfrak{n}$. If $\mathcal{H} \cap \mathfrak{v} \neq 0$ or $\mathcal{H} \cap \mathfrak{z} \neq 0$, then

(i) $\mathcal{H}$ can be written as the orthogonal direct sum $\mathcal{H}=(\mathcal{H} \cap \mathfrak{v}) \oplus(\mathcal{H} \cap \mathfrak{z})$,

(ii) If $j(Z)$ leaves invariant $\mathcal{H} \cap \mathfrak{v}$ for some nonzero element $Z$ of $\mathcal{H} \cap \mathfrak{z}$, then $\mathcal{H}$ is a totally geodesic subalgebra of $\mathfrak{n}$.

Example 4.4 (iii) is a totally geodesic subalgebra of a singular Lie algebra. It is clear in this example that Lemma 4.13 (i) does not hold. For an almost nonsingular example, let $\mathfrak{n}$ be the Lie algebra given by Example 2.5 (ii). Let $\mathcal{H}$ be the subalgebra with basis $\left\{X_{1}, X_{2}, X_{3}+Z_{4}, Z_{1}\right\}$. It is straightforward to check that this is a totally geodesic subalgebra which cannot be decomposed as in (i) above.

To see that Lemma 4.13 (ii) does not hold in the singular case, see Example 2.5 (iii). Let $\mathcal{H}=\operatorname{span}\left\{X_{2}, Z_{3}, X_{4}+Z_{1}, X_{3}+Z_{4}\right\}$. Then $\mathcal{H} \cap \mathfrak{v}$ has basis $\left\{X_{2}\right\}$ and $\mathcal{H} \cap \mathfrak{z}$ has basis $\left\{Z_{3}\right\}$, and thus $j\left(Z_{3}\right)$ leaves $\mathcal{H} \cap \mathfrak{v}$ invariant. $\mathcal{H}$ is an abelian subalgebra; however, $\mathcal{H}$ is not a totally geodesic subalgebra since $\nabla_{X_{4}+Z_{1}}\left(X_{3}+Z_{4}\right)=-\frac{1}{2} X_{6}$ is not in $\mathcal{H}$.

4.4. Decomposition of the tangent space. Let $H$ be a totally geodesic submanifold of $N$ containing the identity $e$ with $\mathcal{H}=T_{e} H \subseteq \mathfrak{n}$. For any element $\xi \in \mathcal{H}$, using the definitions of $\mathcal{H}_{+}^{\xi}, \mathcal{H}_{-}^{\xi}$ and $\mathcal{H}_{0}^{\xi}$ above, we characterize the decomposition of $\mathcal{H}$ in the following results.

Lemma 4.14. Let $H$ be a totally geodesic submanifold of a 2-step nilpotent Lie group $N$ containing e with $\mathcal{H}=T_{e} H \subseteq \mathfrak{n}$. If $0 \neq \xi \in \mathcal{H}$, then we can decompose $\mathcal{H}$ as $\mathcal{H}=\mathcal{H}_{+}^{\xi} \oplus \mathcal{H}_{-}^{\xi} \oplus \mathcal{H}_{0}^{\xi}$.

Proof. Since $H$ is totally geodesic, by Lemma 4.11, $R_{\xi}(\sigma) \in \mathcal{H}$ for any $\xi, \sigma \in \mathcal{H}$. Further, since $R_{\xi}$ is symmetric, $\mathcal{H}$ has an orthonormal basis of eigenvectors of $R_{\xi}$. We can write that basis as 
$\left\{\zeta_{1}, \ldots, \zeta_{r}, \rho_{1}, \ldots, \rho_{s}, \sigma_{1}, \ldots, \sigma_{l}\right\}$ with $\zeta_{i} \in \mathcal{H}_{-}^{\xi}, \rho_{i} \in \mathcal{H}_{+}^{\xi}$ and $\sigma_{i} \in$ $\mathcal{H}_{0}^{\xi}$.

Corollary 4.15. Let $H$ be a totally geodesic submanifold of $N$ containing e with $\mathcal{H}=T_{e} H \subseteq \mathfrak{n}$. If $\mathcal{H} \cap \mathfrak{v} \neq\{0\}$ or $\mathcal{H} \cap \mathfrak{z} \neq\{0\}$, then

(i) In the case $0 \neq X \in \mathcal{H} \cap \mathfrak{v}$,

$$
\mathcal{H}_{-}^{X} \subseteq\{V \in \mathcal{H} \cap \mathfrak{v} \mid[X, V] \neq 0\}
$$$$
\mathcal{H}_{+}^{X} \subseteq\{Z \in \mathcal{H} \cap \mathfrak{z} \mid j(Z) X \neq 0\}
$$$$
\mathcal{H}_{0}^{X}=\left\{\sigma_{\mathfrak{v}}+\sigma_{\mathfrak{z}} \in \mathcal{H} \mid \sigma_{\mathfrak{v}} \in \mathfrak{v}, \sigma_{\mathfrak{z}} \in \mathfrak{z} \text { where }\left[X, \sigma_{\mathfrak{v}}\right]=0 \text { and } j\left(\sigma_{\mathfrak{z}}\right) X=0\right\} \text {, }
$$

and $\mathcal{H}=\mathcal{H}_{-}^{X} \oplus \mathcal{H}_{+}^{X} \oplus \mathcal{H}_{0}^{X}$.

(ii) In the case $0 \neq Z \in \mathcal{H} \cap \mathfrak{z}$,

$$
\begin{aligned}
\mathcal{H}_{-}^{Z} & =\{0\} \\
\mathcal{H}_{+}^{Z} & \subseteq\{V \in \mathcal{H} \cap \mathfrak{v} \mid j(Z) V \neq 0\} \\
\mathcal{H}_{0}^{Z} & =\left\{\sigma_{\mathfrak{v}}+\sigma_{\mathfrak{z}} \in \mathcal{H} \mid \sigma_{\mathfrak{v}} \in \mathfrak{v}, \sigma_{\mathfrak{z}} \in \mathfrak{z} \text { where } j(Z) \sigma_{\mathfrak{v}}=0\right\}, \\
\text { and } \mathcal{H} & =\mathcal{H}_{+}^{Z} \oplus \mathcal{H}_{0}^{Z} .
\end{aligned}
$$

\section{Proof.}

(i) In the case $0 \neq X \in \mathcal{H} \cap \mathfrak{v}, \mathcal{H}_{-}^{X}=\left\{\sigma \in \mathcal{H} \mid R_{X}(\sigma)=\lambda \sigma, \lambda<\right.$ $0\} \subseteq \mathcal{H} \cap \mathfrak{v}$ and $\mathcal{H}_{+}^{X}=\left\{\sigma \in \mathcal{H} \mid R_{X}(\sigma)=\lambda \sigma, \lambda>0\right\} \subseteq \mathcal{H} \cap \mathfrak{z}$ by Lemma 3.1. Then, for $V \in \mathcal{H}_{-}^{X}, R_{X}(V)=\frac{3}{4} j([V, X]) X=$ $\lambda V, \lambda<0$ and $[V, X] \neq 0$ by Lemma 2.2. For $Z \in \mathcal{H}_{+}^{X}$, $R_{X}(Z)=\frac{1}{4}[X, j(Z) X]=\lambda Z, \lambda>0$. Then $j(Z) X \neq 0$ by Lemma 2.2. Next we consider $R_{X}\left(\sigma_{\mathfrak{v}}+\sigma_{\mathfrak{z}}\right)=0$, where $\sigma_{\mathfrak{v}} \in \mathfrak{v}, \sigma_{\mathfrak{z}} \in \mathfrak{z}$. By Lemma 3.2, $\left[X, \sigma_{\mathfrak{v}}\right]=0$ and $j\left(\sigma_{\mathfrak{z}}\right) X=0$. Then $\mathcal{H}_{0}^{X} \subseteq\left\{\sigma_{\mathfrak{v}}+\sigma_{\mathfrak{z}} \in \mathcal{H} \mid \sigma_{\mathfrak{v}} \in \mathfrak{v}, \sigma_{\mathfrak{z}} \in \mathfrak{z}\right.$ where $\left[X, \sigma_{\mathfrak{v}}\right]=0$ and $\left.j\left(\sigma_{\mathfrak{z}}\right) X=0\right\}$. If $\sigma=\sigma_{\mathfrak{v}}+\sigma_{\mathfrak{z}} \in \mathcal{H}$ such that $\left[X, \sigma_{\mathfrak{v}}\right]=0$ and $j\left(\sigma_{\mathfrak{z}}\right) X=0$, then it follows that $R_{X}(\sigma)=0$ by direct calculation. Thus, $\sigma \in \mathcal{H}_{0}^{X}$ and $\mathcal{H}_{0}^{X}=\left\{\sigma_{\mathfrak{v}}+\sigma_{\mathfrak{z}} \in \mathcal{H} \mid \sigma_{\mathfrak{v}} \in \mathfrak{v}, \sigma_{\mathfrak{z}} \in \mathfrak{z}\right.$ where $\left[X, \sigma_{\mathfrak{v}}\right]=0$ and $\left.j\left(\sigma_{\mathfrak{z}}\right) X=0\right\}$. Finally, $\mathcal{H}=\mathcal{H}_{-}^{X} \oplus \mathcal{H}_{+}^{X} \oplus \mathcal{H}_{0}^{X}$.

(ii) In the case $0 \neq Z \in \mathcal{H} \cap \mathfrak{z}, \mathcal{H}_{-}^{Z}=\{0\}$ and $\mathcal{H}_{+}^{Z}=\{\sigma \in$ $\left.\mathcal{H} \mid R_{Z}(\sigma)=\lambda \sigma, \lambda>0\right\} \subseteq \mathcal{H} \cap \mathfrak{v}$ by Lemma 3.1. For $V \in \mathcal{H}_{+}^{Z}$, $R_{Z}(V)=-\frac{1}{4} j(Z)^{2} V=\lambda V, \lambda>0$; therefore, $j(Z) V \neq 0$. Then we consider $R_{Z}\left(\sigma_{\mathfrak{v}}+\sigma_{\mathfrak{z}}\right)=0$ and $\sigma_{\mathfrak{v}} \in \mathfrak{v}, \sigma_{\mathfrak{z}} \in \mathfrak{z}$. We calculate 
$R_{Z}\left(\sigma_{\mathfrak{v}}+\sigma_{\mathfrak{z}}\right)=-\frac{1}{4} j(Z)^{2} \sigma_{\mathfrak{v}}$ resulting in $\mathcal{H}_{0}^{Z} \subseteq\left\{\sigma_{\mathfrak{v}}+\sigma_{\mathfrak{z}} \in \mathcal{H} \mid \sigma_{\mathfrak{v}} \in\right.$ $\mathfrak{v}, \sigma_{\mathfrak{z}} \in \mathfrak{z}$ where $\left.j(Z) \sigma_{\mathfrak{v}}=0\right\}$, by Lemma 2.2. It is obvious that, if $j(Z) \sigma_{\mathfrak{v}}=0$, then $R_{Z}(\sigma)=0$ as well, proving the equality of sets. Thus, $\mathcal{H}_{0}^{Z}=\left\{\sigma_{\mathfrak{v}}+\sigma_{\mathfrak{z}} \in \mathcal{H} \mid \sigma_{\mathfrak{v}} \in \mathfrak{v}, \sigma_{\mathfrak{z}} \in \mathfrak{z}\right.$ where $\left.j(Z) \sigma_{\mathfrak{v}}=0\right\}$ and $\mathcal{H}=\mathcal{H}_{+}^{Z} \oplus \mathcal{H}_{0}^{Z}$.

Remark 4.16. In the case that $j(Z)$ is nonsingular for all $Z \in \mathfrak{z}$, Lemma 4.13 gives the decomposition $\mathcal{H}=(\mathcal{H} \cap \mathfrak{v}) \oplus(\mathcal{H} \cap \mathfrak{z})$. This agrees with Corollary 4.15 above since, in this case, $\mathcal{H}_{-}^{X}=\mathcal{H}_{+}^{Z}=\mathcal{H} \cap \mathfrak{v}$, $\mathcal{H}_{+}^{X}=\mathcal{H}_{0}^{Z}=\mathcal{H} \cap \mathfrak{z}$ and $\mathcal{H}_{0}^{X}=\mathcal{H}_{-}^{Z}=\{0\}$ for all $X \in \mathcal{H} \cap \mathfrak{v}, Z \in \mathcal{H} \cap \mathfrak{z}$.

Note that the decomposition depends on the element of $\mathcal{H}$ that is being considered, and the decompositions will not necessarily be the same for different elements of $\mathcal{H}$.

\section{Example 4.17.}

(i) Let $\mathcal{H}=\mathfrak{n}$ of Example 2.5 (i). Then $\mathcal{H}$ has basis $\left\{X_{1}, X_{2}, X_{3}, X_{4}\right.$, $\left.Z_{1}, Z_{2}\right\}$. Consider the decomposition first with respect to $X_{1}$ : $\mathcal{H}_{-}^{X_{1}}=\operatorname{span}\left\{X_{2}\right\}, \mathcal{H}_{+}^{X_{1}}=\operatorname{span}\left\{Z_{1}\right\}$ and $\mathcal{H}_{0}^{X_{1}}=\operatorname{span}\left\{X_{1}, X_{3}, X_{4}\right.$, $\left.Z_{2}\right\}$. Then with respect to $X_{3}: \mathcal{H}_{-}^{X_{3}}=\operatorname{span}\left\{X_{4}\right\}, \mathcal{H}_{+}^{X_{3}}=$ $\operatorname{span}\left\{Z_{2}\right\}$ and $\mathcal{H}_{0}^{X_{3}}=\operatorname{span}\left\{X_{1}, X_{2}, X_{3}, Z_{1}\right\}$. With respect to $Z_{1}$ : $\mathcal{H}_{+}^{Z_{1}}=\operatorname{span}\left\{X_{1}, X_{2}\right\}$ and $\mathcal{H}_{0}^{Z_{1}}=\operatorname{span}\left\{X_{3}, X_{4}, Z_{1}, Z_{2}\right\}$.

(ii) Consider $\mathfrak{n}$ of Example 2.5 (iii). Let $\mathcal{H}$ be the subalgebra of Example 4.4 (iv). For $\xi=\alpha_{1} X_{5}+\alpha_{2} X_{6}, \mathcal{H}_{-}^{\xi}=\mathcal{H}_{+}^{\xi}=\{0\}$ and $\mathcal{H}_{0}^{\xi}=\mathcal{H}$. As noted above, $\mathcal{H}$ is totally geodesic if and only if $\alpha_{1} \alpha_{3}=-\alpha_{2} \alpha_{4}$.

4.5. Applications of the decomposition. The following lemmas give sufficient conditions for $\mathcal{H}$ to be totally geodesic, generalizing Lemma 4.13. When we have $\mathcal{H} \cap \mathfrak{v} \neq\{0\}$ or $\mathcal{H} \cap \mathfrak{z} \neq\{0\}$, we can say something more about the subset $\mathcal{H} \subseteq \mathfrak{n}$. The first result concerns the existence of an element $Z \in \mathcal{H} \cap \mathfrak{z}$ such that $j(Z)$ is nonsingular on $\pi_{\mathfrak{v}}(\mathcal{H})$.

Lemma 4.18. Let $H$ be a totally geodesic submanifold of $N$ containing e with $\mathcal{H}=T_{e} H \subset \mathfrak{n}$. Suppose there exists $Z \in \mathcal{H} \cap \mathfrak{z}$ such that $j(Z)$ is 
nonsingular on $\pi_{\mathfrak{v}}(\mathcal{H})$. Then, if $\mathcal{H} \cap \mathfrak{v}$ is invariant under $j(Z), \mathcal{H}$ is a totally geodesic subalgebra.

Proof. Since $j(Z)$ is nonsingular on $\pi_{\mathfrak{v}}(\mathcal{H}), \mathcal{H}_{0}^{Z}=\mathcal{H} \cap \mathfrak{z}$, and then $\mathcal{H}_{+}^{Z}=\mathcal{H} \cap \mathfrak{v}=\pi_{\mathfrak{v}}(\mathcal{H})$. Thus, $\mathcal{H}=(\mathcal{H} \cap \mathfrak{v}) \oplus(\mathcal{H} \cap \mathfrak{z})$. Also, since $j(Z)$ is nonsingular and $j(Z)$ keeps $\mathcal{H} \cap \mathfrak{v}$ invariant, for every $Y \in \mathcal{H} \cap \mathfrak{v}$, there exists $X^{*} \in \mathcal{H} \cap \mathfrak{v}$ such that $Y=j(Z) X^{*}$. Then, for any elements $\xi=X+Z_{1}, \sigma=Y+Z_{2} \in \mathcal{H}$ where $X, Y \in \mathcal{H} \cap \mathfrak{v}$ and $Z_{1}, Z_{2} \in \mathcal{H} \cap \mathfrak{z},[\xi, \sigma]=[X, Y]=\left[X, j(Z) X^{*}\right] \in \mathcal{H} \cap \mathfrak{z}$ by Lemma 4.12. Thus, $\mathcal{H}$ is a subalgebra of $\mathfrak{n}$. As previously calculated, $\nabla_{\xi} \sigma=\frac{1}{2}\left([X, Y]-j\left(Z_{2}\right) X-j\left(Z_{1}\right) Y\right)$. Thus, for $\mathcal{H}$ to be totally geodesic, it is necessary for $j\left(Z_{2}\right) X+j\left(Z_{1}\right) Y \in \mathcal{H}$. As above, there exists $X^{*} \in \mathcal{H} \cap \mathfrak{v}$ such that $X=j(Z) X^{*}$ for each $X \in \mathcal{H} \cap \mathfrak{v}$; then, $j\left(Z_{2}\right) X=j\left(Z_{2}\right) j(Z) X^{*}=-4 R\left(X^{*}, Z_{2}\right) Z \in \mathcal{H}$ by Lemma 4.11. Similarly, $j\left(Z_{1}\right) Y \in \mathcal{H}$.

Note that the presence of $Z \in \mathcal{H} \cap \mathfrak{z}$ such that $j(Z)$ nonsingular does not depend on $\mathfrak{n}$ being nonsingular or almost nonsingular. Consider the following examples. In both cases, there exists a nonzero $Z \in \mathcal{H} \cap \mathfrak{z}$ such that $j(Z)$ is nonsingular on $\pi_{\mathfrak{v}}(\mathcal{H})$, but there also exist other nonzero $\widetilde{Z} \in \mathcal{H} \cap \mathfrak{z}$ such that $j(\widetilde{Z})$ is singular on $\mathcal{H}$.

\section{Example 4.19.}

(i) Let $\mathcal{H}$ be a subspace of the 12-dimensional Lie algebra $\mathfrak{n}$ of Example 2.5 (ii) with basis $\left\{X_{1}, X_{2}, X_{5}, X_{6}, Z_{1}, Z_{3}\right\}$. Recall that $\mathfrak{n}$ is almost nonsingular. Let $Z=\alpha_{1} Z_{1}+\alpha_{2} Z_{3}$ with both $\alpha_{1}$ and $\alpha_{2}$ nonzero, and let $X \in \mathcal{H} \cap \mathfrak{v}$. Write $X=\beta_{1} X_{1}+\beta_{2} X_{2}+\beta_{3} X_{5}+$ $\beta_{4} X_{6}$. Then $j(Z) X=\alpha_{1} \beta_{1} X_{2}-\alpha_{1} \beta_{2} X_{1}+\alpha_{2} \beta_{3} X_{6}-\alpha_{2} \beta_{4} X_{5} \neq 0$ unless $\beta_{1}=\beta_{2}=\beta_{3}=\beta_{4}=0$. Thus, $j(Z)$ is nonsingular on $\mathcal{H}$. However, both $j\left(Z_{1}\right)$ and $j\left(Z_{3}\right)$ are singular on $\mathcal{H}$. By direct calculation, it is clear that $\mathcal{H}$ is a totally geodesic subalgebra of $\mathfrak{n}$.

(ii) Consider the singular $\mathfrak{n}$ of Example 2.5 (iii). Let $\mathcal{H}$ be the subspace of $\mathfrak{n}$ with basis $\left\{X_{1}, X_{2}, X_{4}, X_{5}, Z_{1}, Z_{3}\right\}$. For $Z=$ $\alpha_{1} Z_{1}+\alpha_{2} Z_{3}$, both $\alpha_{1}$ and $\alpha_{2}$ nonzero, $j(Z)$ is nonsingular on $\mathcal{H} \cap \mathfrak{v}$ and it preserves $\mathcal{H} \cap \mathfrak{v}$. Again, both $j\left(Z_{1}\right)$ and $j\left(Z_{3}\right)$ are singular on $\mathcal{H}$, and it is clear that $\mathcal{H}$ is a totally geodesic subalgebra. 
Lemma 4.18 provides sufficient but not necessary conditions for $\mathcal{H} \subseteq \mathfrak{n}$ to be totally geodesic. It is not necessary that there exists a $Z \in \mathcal{H} \cap \mathfrak{z}$ such that $j(Z)$ nonsingular on $\pi_{\mathfrak{v}}(\mathcal{H})$ for $\mathcal{H}$ to be a totally geodesic subalgebra. In the following example, for instance, $j(Z)$ is singular for all $Z \in \mathcal{H} \cap \mathfrak{z}$, and $\mathcal{H}$ is a totally geodesic subalgebra of $\mathfrak{n}$.

Example 4.20. Let $\mathcal{H}$ be a subspace of $\mathfrak{n}$ the 12 -dimensional algebra of Example 2.5 (ii) with basis $\left\{X_{1}, X_{2}, X_{3}, X_{5}, X_{6}, Z_{1}, Z_{3}\right\}$. Note that $X_{3} \in \operatorname{ker} j(Z)$ for all $Z \in \mathcal{H}$, so all $j(Z)$ are singular. However, it can be verified that $\mathcal{H}$ is a totally geodesic subalgebra of $\mathfrak{n}$ by a straightforward calculation.

To consider the case where $\mathcal{H}$ may not have a nonsingular $Z$ as required by Lemma 4.18, we consider the projections of $\mathcal{H}$ onto $\mathfrak{v}$ and $\mathfrak{z}$.

Proposition 4.21. Suppose that $\mathcal{H}$ is a subalgebra of a 2-step nilpotent metric Lie algebra $\{\mathfrak{n},\langle\rangle$,$\} . If j(Z) X \in \mathcal{H}$ for all $Z \in \pi_{\mathfrak{z}}(\mathcal{H})$ and all $X \in \pi_{\mathfrak{v}}(\mathcal{H})$, then the subalgebra $\mathcal{H}$ is totally geodesic. Further, if $\mathcal{H} \cap \mathfrak{z}=\{0\}$, then $\mathcal{H}$ is an abelian flat subalgebra of $\mathfrak{n}$.

Proof. Let $\xi, \sigma \in \mathcal{H}$. Since $\xi, \sigma \in \mathfrak{n}$, we write $\xi=X+Z_{1}, \sigma=Y+Z_{2}$ where $X, Y \in \mathfrak{v}$ and $Z_{1}, Z_{2} \in \mathfrak{z}$. As above, $\nabla_{\xi} \sigma=\frac{1}{2}\left([X, Y]-j\left(Z_{2}\right) X-\right.$ $\left.j\left(Z_{1}\right) Y\right)$. Since $\mathcal{H}$ is a subalgebra, $[\xi, \sigma]=[X, Y] \in \mathcal{H}$ and, since $j(Z) X \in \mathcal{H}$ for all $Z \in \pi_{\mathfrak{z}}(\mathcal{H})$ and all $X \in \pi_{\mathfrak{v}}(\mathcal{H})$, it follows that $j\left(Z_{2}\right) X, j\left(Z_{1}\right) Y \in \mathcal{H}$. Thus, $\nabla_{\xi} \sigma \in \mathcal{H}$ for all $\xi, \sigma \in \mathcal{H}$ and $\mathcal{H}$ totally geodesic.

In the case that $\mathcal{H} \cap \mathfrak{z}=\{0\}$, the result follows from Proposition 4.8.

Note that the hypothesis of Lemma 4.21 is actually stronger than necessary. Observe that it is not necessary that $j(Z) X \in \mathcal{H}$ for all $Z \in \pi_{\mathfrak{z}}(\mathcal{H})$ and $X \in \pi_{\mathfrak{v}}(\mathcal{H})$. Example 4.7 is one such abelian flat example. The following example is an additional example of a totally geodesic subalgebra that is neither flat nor abelian and in which there exists a $Z \in \pi_{\mathfrak{z}}(\mathcal{H})$ such that $j(Z) X \notin \mathcal{H} \cap \mathfrak{v}$ for some $X \in \pi_{\mathfrak{v}}(\mathcal{H})$.

Example 4.22. Let $\mathcal{H}=\operatorname{span}\left\{X_{2}+Z_{2},-X_{3}+Z_{1}, X_{4}, X_{5}, X_{6}, Z_{3}, Z_{4}\right\}$ be a subalgebra of the Lie algebra $\mathfrak{n}$ of Example 2.5 (iii). Note that 
$j\left(Z_{1}\right) X_{2}=j\left(Z_{2}\right) X_{3}=-X_{1} \notin \mathcal{H}$, however direct calculation shows that $\mathcal{H}$ is totally geodesic.

4.6. Proof of Theorem 4.10. Below is the proof of the result stated in Theorem 4.10.

Proof. Since $\mathcal{H}$ is totally geodesic, $\nabla_{X} Z=-\frac{1}{2} j(Z) X \in \mathcal{H}$ for all $X \in \mathcal{H} \cap \mathfrak{v}$ and all $Z \in \mathcal{H} \cap \mathfrak{z}$. Thus, $\mathcal{H} \cap \mathfrak{v}$ is invariant under $j(Z)$ for all $Z \in \mathcal{H} \cap \mathfrak{z}$. If either $\mathcal{H} \cap \mathfrak{z}=\{0\}$ or $\mathcal{H} \cap \mathfrak{v}=\{0\}$, then by Proposition 4.8, $\mathcal{H}$ is abelian and flat.

Now consider the cases $\mathcal{H} \cap \mathfrak{z} \neq\{0\}$ and $\mathcal{H} \cap \mathfrak{v} \neq\{0\}$. By Corollary $4.15, \mathcal{H}=\mathcal{H}_{+}^{Z} \oplus \mathcal{H}_{0}^{Z}$ for any $Z \in \mathcal{H} \cap \mathfrak{z} ; \mathcal{H}_{0}^{Z}$ is always nonzero since $Z \in \mathcal{H}_{0}^{Z}$ for any $Z \in \mathcal{H} \cap \mathfrak{z}$. Suppose $\mathcal{H}_{+}^{Z}=\{0\}$ for all $Z \in \mathcal{H} \cap \mathfrak{z}$. Then $j(Z) X=0$ for all $Z \in \mathcal{H} \cap \mathfrak{z}$ and all $X \in \mathcal{H} \cap \mathfrak{v}$. Assume $\mathcal{H}$ is not abelian. That is, there exist $\xi=X+Z_{1}, \sigma=Y+Z_{2} \in \mathcal{H}$ such that $[\xi, \sigma]=[X, Y]=Z \neq 0$ where $Z \in \mathcal{H} \cap \mathfrak{z}$ since $\mathcal{H}$ a subalgebra. Then $0 \neq|Z|^{2}=\langle[X, Y], Z\rangle=\langle j(Z) X, Y\rangle$. But $j(Z) X=0$ for all $X, Z \in \mathcal{H}$, so this is a contradiction. Thus, if $\mathcal{H}_{+}^{Z}=\{0\}$, (i) holds, namely, $\mathcal{H}$ is abelian and flat. If $\mathcal{H}_{+}^{Z}$ is nonzero for some $Z \in \mathcal{H} \cap \mathfrak{z}$, then there exists an $X \in \mathcal{H} \cap \mathfrak{v}$ such that $j(Z) X \neq 0$. Since $\mathcal{H}$ is totally geodesic, $\nabla_{X} Z=-\frac{1}{2} j(Z) X \in \mathcal{H}$ is nonzero and therefore $\mathcal{H}$ is not flat and abelian and (ii) holds.

Acknowledgments. The authors would like the thank the referee for detailed and helpful comments and suggestions.

\section{REFERENCES}

1. R. DeCoste, Closed geodesics on compact nilmanifolds with Chevalley rational structure, Manuscr. Math. 127 (2008), 309-343.

2. R. DeCoste, L. DeMeyer and M. Mast, Heisenberg-like Lie algebras, J. Lie Theor. 21 (2011), 711-727.

3. L. DeMeyer, Closed Geodesics in Compact Nilmanifolds, Manuscr. Math. 105 (2001), 283-310.

4. P. Eberlein, Geometry of 2-step nilpotent groups with a left invariant metric, I, Ann. Scient. Ecole Norm. Sup. 5 (1994), 611-660.

5. , Geometry of two-step nilpotent groups with a left invariant metric II, Trans. AMS, 343, 805-828 (1994).

6. , Left invariant geometry of Lie groups, Cubo 1 (2004), 427-510. 
7. R. Gornet and M. Mast, The length spectrum of Riemannian two-step nilmanifolds, Ann. Sci. Ecole Norm. Sup. 2 (2000), 181-209.

8. A. Kaplan, Riemannian nilmanifolds attached to Clifford modules, Geom. Ded. 11 (1981), 127-136.

9. , On the geometry of groups of Heisenberg type, Bull. Lond. Math. Soc. 15 (1983), 35-42.

10. J.M. Lee, Riemannian manifolds: An introduction to curvature, Grad. Texts Math. 176, Springer-Verlag, New York, 1997.

11. K. Lee and K. Park, Smoothly closed geodesics in 2-step nilmanifolds, Indiana Univ. Math. J. 45 (1996), 1-14.

12. M. Mast, Closed geodesics in 2-step nilmanifolds, Indiana Univ. Math. J. 43 (1994), 885-911.

Department of Mathematics and Computer Science, Wheaton College, NORTON, MA 02766

Email address: decoste_rachelle@wheatoncollege.edu

Department of Mathematics, Central Michigan University, Mount PleasANT, MI 48858

Email address: lisa.demeyer@cmich.edu 